\title{
Avaliação da influência do sistema vestibular no equilíbrio de adultos jovens através de posturografia dinâmica foam-laser e plataforma de força
}

\author{
Evaluation of the influence of vestibular system in the \\ young adults balance through dynamic posturografy \\ foam-laser and platform of power
}

\author{
Eduardo Alexandre Loth ${ }^{1}$; Ângela Garcia Rossi ${ }^{2}$; \\ Priscila Cristina Cappellesso ${ }^{3}$; Adriano Polican Ciena ${ }^{4}$
}

\begin{abstract}
Resumo
Uma das tarefas mais importantes do sistema do controle postural humano é o equilíbrio do corpo sobre a pequena base de apoio fornecida pelos pés. Como um sensor de gravidade, o sistema vestibular é uma das ferramentas mais importantes do sistema nervoso no controle da postura. O presente estudo teve como objetivo estudar a influencia do sistema vestibular no equilíbrio de adultos jovens, por meio da Posturografia Dinâmica Foam-laser (FLP). Para a realização do estudo, foram convidados 30 voluntários adultos jovens, acadêmicos do curso de educação física da UFSM, que foram submetidos à avaliação do controle postural através de FLP e plataforma de força simultaneamente, durante o Teste de Organização Sensorial (TOS) I e V. Os resultados revelaram que houve moderado e forte índice de correlação entre os valores obtidos nos TOS I e V e a área de deslocamento do centro de pressão (CP) registrada pela plataforma de força. Conclui-se que a FLP é um método muito útil para avaliação do equilíbrio, e seus resultados demonstraram boa correlação com a área de deslocamento do CP.

Palavras- chave: Posturografia Dinâmica Foam-Laser. Equilíbrio. Sistema vestibular.
\end{abstract}

\begin{abstract}
One of the most important tasks for the human postural control system is to maintain body balance on a small support base provided by the feet. As a gravity sensor, the vestibular system is one of the most important nervous system tools in posture control. The objective of this work was to study the influence of the vestibular system on the young adults' balance control through Dynamic Posturografy Foam-laser (FLS). Participated in the study 30 young adults from the UFSM's physical education undergraduate course, who volunteered to go under postural control evaluation through the FLP and platform of power, simultaneously, during the Sensorial Organization Test (TOS) I and V. Results showed that there was a
\end{abstract}

1 Fisioterapeuta, Mestre pelo Programa de Pós-graduação em Distúrbios da Comunicação Humana da UFSM /RS e docente e Coordenador do Curso de Fisioterapia da UNIOESTE - campus de Cascavel/PR. E-mail: alefisio@unioeste.br.

2 Fonoaudióloga, Doutora em Distúrbios da Comunicação Humana pela UNIFESP e docente do Programa de Mestrado em Distúrbios da Comunicação Humana da UFSM/RS.

3 Fisioterapeuta, pós-graduanda em Fisioterapia Geriátrica pela Universidade Estadual do Oeste do Paraná - UNIOESTE campus de Cascavel-PR.

${ }^{4}$ Graduado em Fisioterapia, pela Universidade Estadual de Londrina-UEL, Especialista em Fisioterapia Ortopédica e Traumatológica pela Universidade Estadual do Oeste do Paraná - UNIOESTE campus Cascavel-PR- Docente do Curso de Biomedicina e Enfermagem da Universidade Paranaense- UNIPAR.

Semina: Ciências Biológicas e da Saúde, Londrina, v. 29, n. 1, p. 57-64, jan./jun. 2008 
moderate and strong rate of correlation between TOS I and V values and the area of displacement of the pressure center (CP) registered by the platform of power. They also showed that the FLP is a very useful method for balance evaluation, showing a good correlation with the $\mathrm{CP}$ displacement area .

Keywords: Dynamic Posturografy Foam-Laser, balance, vestibular system.

\section{Introdução}

O sistema nervoso central (SNC), integrado com os sistemas vestibular, visual e proprioceptivo, recebe as informações do meio externo fazendo com que o corpo mantenha-se em equilíbrio, executando movimentos em relação ao corpo e ao espaço (VIEIRA, 2006; MAKI; MCILROY, 1996; HORAK; NASHNER, 1996).

A orientação postural está relacionada à manutenção da posição dos segmentos corporais em relação aos outros segmentos e ao meio ambiente. O equilíbrio postural está relacionado com as forças internas e externas que agem no corpo durante as ações motoras (BARELA, 2000).

O equilíbrio é a integração sensório-motora que garante a manutenção da postura. Manter a postura ereta estável é uma tarefa complexa, pois a mesma deve ser mantida sob a ação de forças externas que desestabilizam o corpo e sobre uma base de apoio relativamente pequena fornecida pelos pés. Para que o corpo humano mantenha-se estável na postura ereta, o sistema de controle postural deve organizar suas ações de forma eficiente, por meio da interação dos sistemas nervoso, sensorial e motor (WINTER, 1990; HORAK; MACPHERSON, 1996; MAK; MCILROY, 1996; HORAK; SHUPERT; MIRKA, 1989).

O sistema motor é responsável pela ativação correta e adequada de músculos para realização dos movimentos. O sistema nervoso central integra informações provenientes do sistema sensorial para, então, enviar impulsos nervosos aos músculos que geram respostas neuromusculares (DUARTE; ZATSIORSKY, 2002).

Ao estudar postura corporal automaticamente estuda-se o sistema de equilíbrio postural, pois existe uma relação de dependência entre ambos. O reflexo de endireitamento utilizado para a manutenção da postura se faz importante para que se entenda a complexidade da postura corporal, pois se trata de um trabalho integrado e simultâneo. Em algumas situações, caso essa integração seja interrompida, poderá ocorrer algum desequilíbrio. É o que ocorre quando perdemos a seqüência lógica de passos em uma caminhada, quando ocorre erro nos passos de uma dança em relação ao ritmo,e isso é devido à interrupção momentânea no circuíto integrado desses reflexos posturais. Os reflexos de endireitamentos labirínticos, que atuam sobre a cabeça, pescoço, corpo e os nervos ópticos, situamse na parte ventral do mesencéfalo, localizados em frente ao terceiro par de nervos cranianos. Pouco se sabe a respeito de que forma esses reflexos contribuem para proporcionar uma postura corporal ereta e equilibrada, porém sabe-se o quanto eles são importantes (BANKOFF et al., 1992; DI GRAZIA, 2003).

Como um sensor de gravidade, o sistema vestibular é uma das ferramentas mais importantes do sistema nervoso no controle da postura. Além dele, os impulsos captados no órgão da visão e nos receptores proprioceptivos, juntamente com uma resposta muscular reflexa, também são responsáveis pela manutenção do equilíbrio e do controle postural (HERDMAN, 2002).

A avaliação do equilíbrio representa um grande desafio para a ciência, sobretudo por advir de uma função conjunta de vários sistemas complexos. Neste contexto, objetivou-se através desta pesquisa avaliar a influência do sistema vestibular sobre o equilíbrio de adultos jovens através da Posturografia Dinâmica Foam-laser e plataforma de força. 


\section{Materiais e métodos}

Este estudo foi desenvolvido em conjunto com o laboratório de Biomecânica da Universidade Federal de Santa Maria (UFSM) após a aprovação pelo comitê de ética da Universidade Estadual do Oeste do Paraná (UNIOESTE), conforme registro número 19366/2006 e aprovado pelo parecer 226/2006.

A pesquisa foi realizada no laboratório de biomecânica da UFSM, no período de 18 a 26 de março de 2006. Para tanto foram recrutados 30 (19 homens e 11 mulheres), acadêmicos do Curso de Educação Física da UFSM, com idade média de $21,17 \pm 1,45$ anos, que caracterizou a população amostral como estratificada probabilística simples.

Os critérios de inclusão foram: acadêmicos do Curso de Educação Física da Universidade Federal de Santa Maria/RS, com idade entre 18 a 30 anos, os quais concordaram em assinar o Termo de Consentimento Livre e Esclarecido. Foram excluídos do estudo os indivíduos que apresentaram amputação de algum membro, doença neurológica conhecida, traumas ortopédicos agudos e deficientes visuais graves.

Em seguida, os voluntários foram submetidos à avaliação do controle postural pelo método de Posturografia Dinâmica Foam-Laser (FLP) proposto por Castagno (1994) e plataforma de força (OR6-6, Advanced Mechanical Tecnology Incorporation, USA), simultaneamente. Para tanto, cada participante foi posicionado dentro de uma cabine de $1 \mathrm{~m}^{2}$, com $2 \mathrm{~m}$ de altura, posicionada sobre uma plataforma de força, recoberta com um tecido constituído de listras laranjas e beges de $10 \mathrm{~cm}$ e uma almofada de espuma de densidade média com $10 \mathrm{~cm}$ de espessura (figura 1).

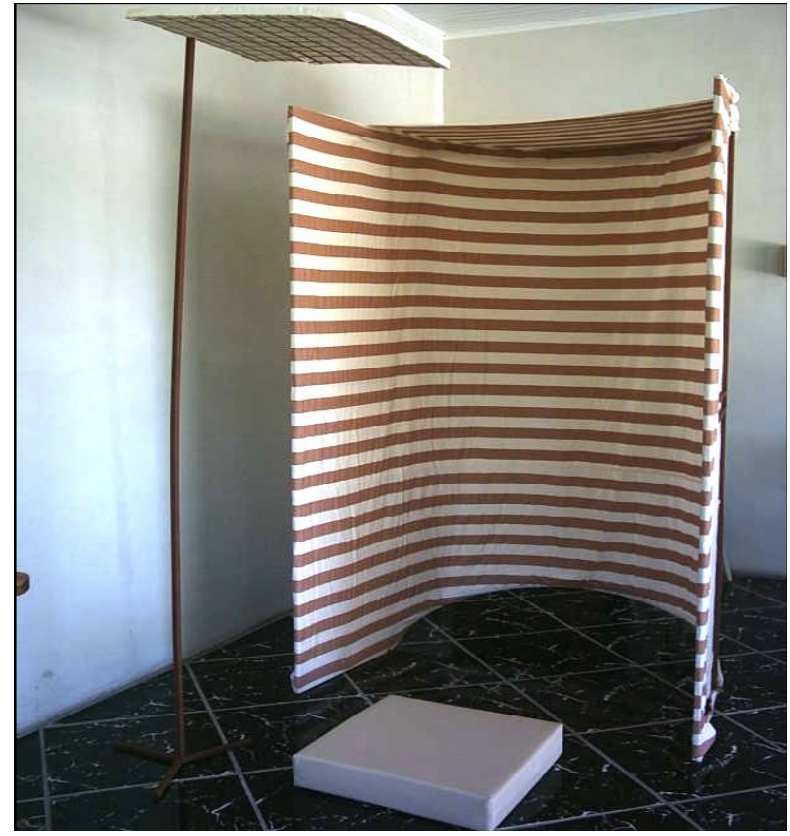

Figura 1. Cabine da Foam-laser Dynamic Posturography proposto por Castagno (1994).

Na região posterior do voluntário, foi acoplado um cinto de Nylon, ajustável a cintura do paciente, contendo uma caneta que emitia um feixe de laser, direcionado para uma escala em centímetros fixada acima da cabine, no plano horizontal (figuras $2 \mathrm{e}$ 3). O feixe de laser permitiu avaliar o deslocamento antero-posterior durante os Testes de Organização Sensorial (TOS), que é um método de avaliar o equilíbrio corporal no qual os voluntários são submetidos a avaliação do equilíbrio na posição em pé, em situações diversas .

Para obtenção dos dados, cada participante foi submetido à avaliação do controle postural nos TOS I e $\mathrm{V}$, e nela era medida a distância em $\mathrm{cm}$ entre a fonte luminosa (caneta laser) fixada no voluntário e o anteparo disposto acima da cabine de tecido (papel centimetrado). Essa distância representou a medida entre as letras $\mathrm{C}$ e L e o deslocamento anteroposterior máximo do avaliado, por meiodo feixe de luz no papel centimetrado, durante a realização dos TOS. Esta distância representou a medida entre as letras $\mathrm{C}$ e $\mathrm{A}$ da fórmula contida na figura 4 . 


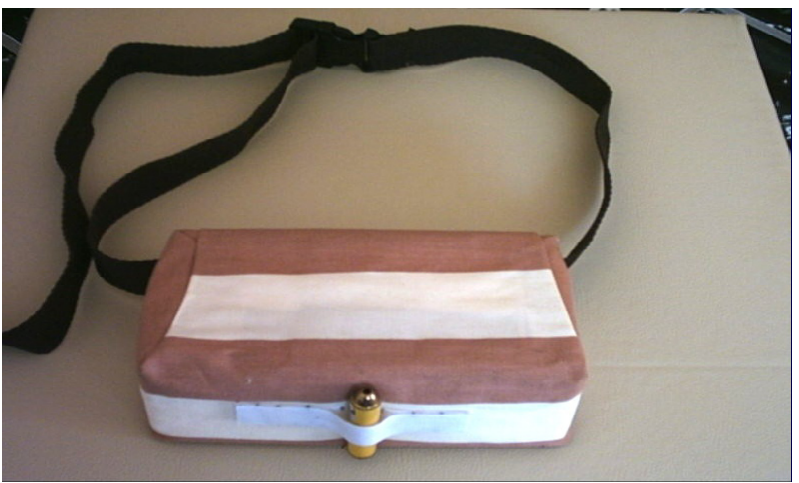

Figura 2. Cinto confeccionado em tecido de Nylon e isopor, com encaixe nas extremidades ajustáveis ao nível da cintura do participante, com caneta de feixe de laser acoplado.

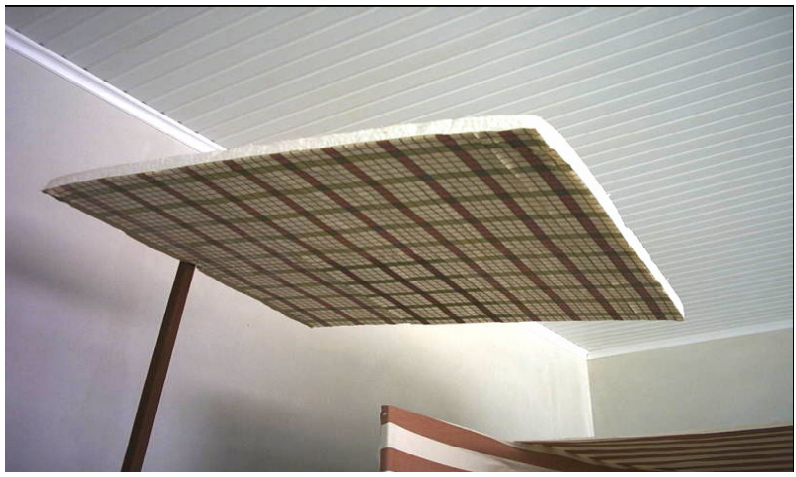

Figura 3. Papel com escala em centímetros fixada acima da cabine, a fim de anotar o maior deslocamento em centímetros para realização cálculo da FLP.

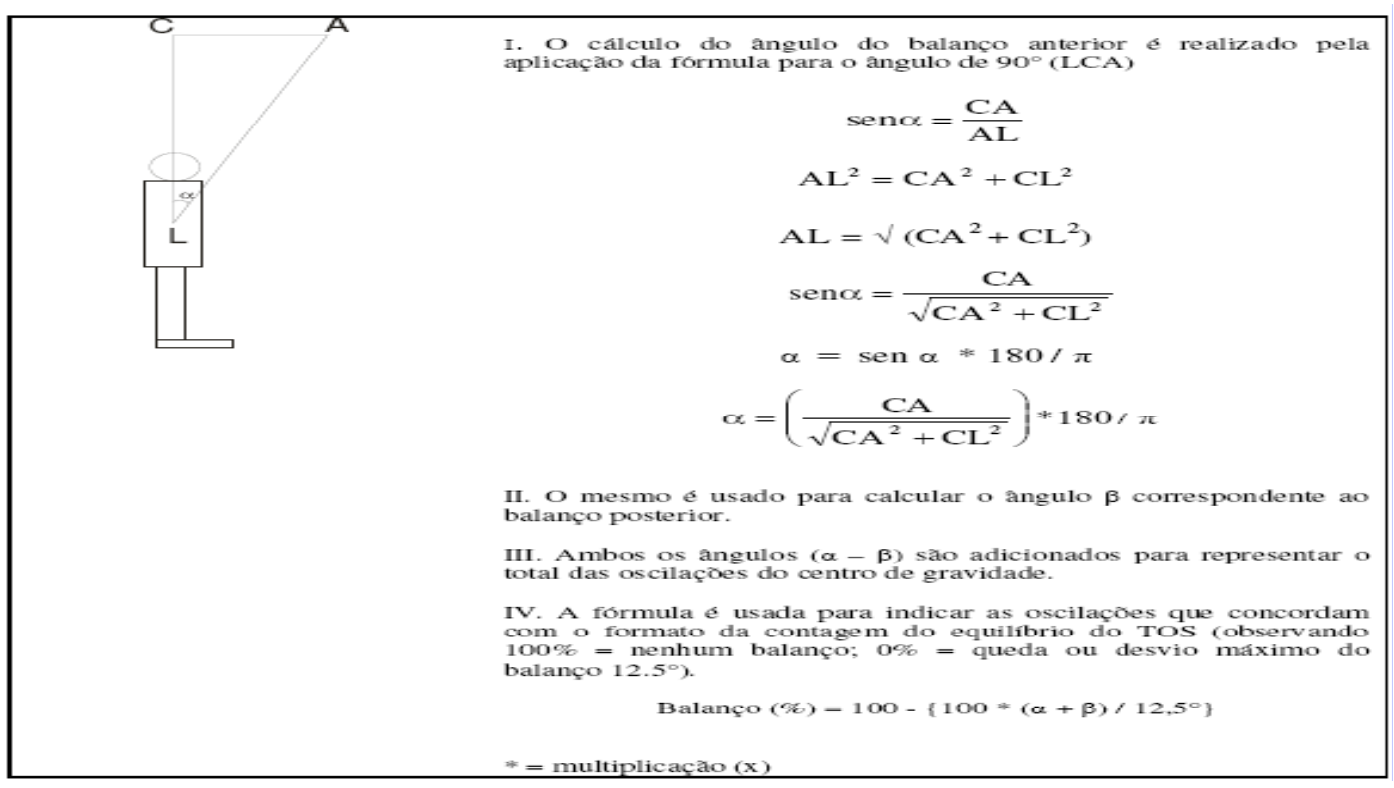

Figura 4. Fórmula matemática para o cálculo do ângulo de oscilação corporal proposta pela técnica da Posturografia Dinâmica "Foam-laser". Fonte: (Castagno, 1994).

O TOS I, foi realizado com os participantes na posição ortostática sobre a superfície rígida da plataforma de força, pés posicionados a largura dos ombros, braços ao longo do corpo, olhos abertos com olhar fixado a frente em uma das listras da FLP, a cerca de $80 \mathrm{~cm}$ de distância. O TOS V foi realizado com o voluntário na posição anterior, mas sobre uma almofada de espuma de $10 \mathrm{~cm}$ de espessura de densidade média, com a finalidade de atenuar a propriocepção do examinado. Cada TOS foi realizado durante o tempo de 20 segundos de registro.
Para análise dos dados registrados na plataforma de força que operou a $100 \mathrm{~Hz}$, utilizou-se o programa MATLAB $^{\circledR}$-7.4, para calcular a área de deslocamento do centro de pressão em $\mathrm{cm}^{2}$. Os resultados da FLP foram obtidos por meio da fórmula matemática para cálculos de ângulos de oscilações apresentados. Para avaliar o grau de associação entre os resultados, foi utilizado o índice de correlação de Spearman, adotando-se o nível de significância de $5 \%$ com p> 0,05 através do software GraphPad Prism ${ }^{\circledR}$ versão 3.0. 


\section{Resultados}

A analise dos resultados obtidos por meio da FLP indica que a média dos escores da amostra declinaram de 88,27 pontos para 68.83 pontos conforme disposto na tabela 1 .

Após a análise dos resultados obtidos através do registro da plataforma de força e expressos em área por centímetros quadrados $\left(\mathrm{cm}^{2}\right)$, observou-se que a média obtida no TOS I e V foi de $0,88 \mathrm{~cm}^{2}$ e 3,56 , respectivamente.
Após realizar a análise de correlação para avaliar o grau de associação dos resultados obtidos, entre as condições da FLP com a área de deslocamento do $\mathrm{CP}$ registrada pela Plataforma de Força, observouse moderada correlação no TOS I, com r= -0,60.

No TOS V, observou-se forte correlação entre o resultado do exame da FLP e plataforma de força, com $r=-0,70$.

Tabela 1. Apresentação dos resultados através de médias, desvio padrão e valores máximo e mínimo em percentual (\%), obtidos no exame de Posturografia Dinâmica Foam-Laser, do grupo estudado.

\begin{tabular}{lcc}
\hline & TOS I & TOS V \\
\hline Média & 88,27 & 68,83 \\
Desvio-padrão & 4,60 & 10,86 \\
Máximo & 95,51 & 86,91 \\
Mínimo & 73,85 & 41,96 \\
Valor de referência & 90 & 60 \\
\hline
\end{tabular}

Fonte: Autores.

Tabela 2. Apresentação dos resultados através de médias, desvio padrão e valores máximo e mínimo da área de deslocamento do $\mathrm{CP} \mathrm{em} \mathrm{cm}^{2}$ registrados pela plataforma de força.

\begin{tabular}{lcc}
\hline & TOS I & TOS V \\
\hline Média & 0,88 & 3,56 \\
Desvio-padrão & 0,64 & 1,55 \\
Máximo & 1,98 & 8,10 \\
Mínimo & 0,17 & 0,89 \\
\hline
\end{tabular}

Fonte: Autores.

Tabela 3. Apresentação dos índices de correlação dos resultados obtidos através da realização da FLP e a média da área do deslocamento do $\mathrm{CP} \mathrm{em} \mathrm{cm}^{2}$ obtida, simultaneamente, com a Plataforma de Força. O sinal negativo e o asterisco indicam, respectivamente, que a correlação é inversamente proporcional e o valor de p. encontrado é estatisticamente significante, com nível de significância de 5\%.

\begin{tabular}{lcc}
\hline Procedimento & TOS I & TOS V \\
\hline Posturografia Dinâmica & 88,27 & 68,83 \\
Plataforma de força & 0.88 & 3.56 \\
Índice de correlação & -0.60 & -0.70 \\
Valor de p. & $0.0004^{*}$ & $0.0001^{*}$ \\
\hline
\end{tabular}

Fonte: Autores. 


\section{Discussão}

Aanálisedecorrelaçãodos resultadosobtidospelos procedimentos para a avaliação do controle postural no estudo, permitiu observar que houve moderado e forte índice de correlação negativa (inversamente proporcional) no TOS I e V respectivamente, entre os resultados da FLP e plataforma de força. Essa correlação é assim expressa: quanto maior a área de deslocamento do $\mathrm{CP}$ registrada pela plataforma de força, maior também a oscilação detectada na FLP. Como os escores da FLP são considerados valores em percentuais, e que, na ausência total de oscilação corporal, o escore obtido é $100 \%$, decrescendo de acordo com o aumento de oscilações. Assim, quanto maior for à área de deslocamento do $\mathrm{CP}$ na plataforma de força, menor será o escore obtido na FLP, o que determina índice de correlação negativa entre as análises.

Nashner (1993) relata que a disfunção vestibular pode ser identificada no exame de posturografia computadorizada por meio do aumento da instabilidade corporal nas condições impostas pelo TOS I e V. Do mesmo modo, Ledin e Odkvist (1993) descrevem que as anormalidades do sistema vestibular são observados por resultados anormais no TOS V, que indicam inabilidade para o uso de informações vestibulares. Esses autores relataram a utilidade do EquiTest no estudo dos efeitos da idade sobre a função vestibular e avaliação dos métodos de reabilitação labiríntica.

No presente estudo, observa-se aumento da oscilação corporal no TOS V em relação ao TOS I, porém, a média dos escores foi acima do valor de referência sugerido pelo idealizador da FLP.

Nashner (1971) comenta que o controle da postura ereta envolve a integração dos sistemas sensoriais múltiplos, os quais fornecem informações convergentes e redundantes que permitem o controle flexível do equilíbrio.

Nesse sentido, Sage (1984), considera que a função mais importante do aparelho vestibular é fornecer informação acerca do posicionamento e movimentação da cabeça com relação ao ambiente. Em virtude dos receptores vestibulares estarem restritos à cabeça, as informações vestibulares necessitam da combinação das informações da posição do tronco para a discriminação da posição de um segmento corporal em relação ao outro.

Castagno (1994), em seus estudos, idealizou um método de avaliação do controle postural, análogo ao Equitest of Neurocom Int Inc, Clackams, Oregon, USA, que também realiza o Teste de Organização Sensorial denominado Foam-laser Posturography Dinamic. O mesmo autor relata que, devido ao alto custo do Equitest, fato que inviabiliza seu uso na maioria das instituições, a FLP representa um método de baixo custo e confiável para realização do TOS. Esse método é capaz de quantificar os distúrbios do equilíbrio corporal, permitindo caracterizaros efeitos da idade sobre a função vestibular, e é especialmente eficaz para avaliar a evolução da enfermidade e a efetividade dos métodos de reabilitação labiríntica empregados.

Corroborando com o autor descrito acima, Lobo (2002), salientou a importância deste método como ferramenta de avaliação do equilíbrio corporal, em seus estudos para avaliar os benefícios de um programa de reabilitação vestibular em idosas institucionalizadas, relizado em em Porto Alegre RS.

O método de FLP, utilizado nesse estudo, também serviu de ferramenta diagnóstica para Yassue et al. (2006), que encontraram alterações no equilíbrio de idosos portadores de diabetes mellitus por meio da FLP. Os autores sugeriram que esta técnica constitui uma ferramenta muito sensível para avaliação do equilíbrio em diabéticos.

No entanto, a FLP representa um método quanti/ qualitativo, de grande valia para avaliação do controle postural, e deve ser explorado por identificar a influência dos sistemas que são responsáveis no controle postural em um distúrbio do equilíbrio, servindo como ferramenta no auxílio ao diagnóstico destes distúrbios. Cabe ressaltar que em um país de 
população como o Brasil, onde os recursos são raros e a medicina em muitos locais ainda é arcaica, a FLP é de fácil aquisição, baixo custo e tem múltiplas aplicações, podendo ser facilmente transportado de um local para outro em avaliações domiciliares.

Do mesmo modo, Voorhess (1989) relata que a FLP não pode detectar as latências que são úteis no diagnóstico neurológico, tão pouco executar análise se a estratégia do quadril ou do tornozelo está sendo utilizada; nem marcar o deslocamento do centro de gravidade. Entretanto, é uma técnica simples, barata e útil que produz a análise do teste de organização sensorial, muito comparável aos obtidos com o EquiTest $^{\circledR}$.

No entanto, ao realizar um exame utilizando a FLP, o examinador não deverá ter a pretensão de obter dados sobre a cinética do controle postural, como os obtidos pela plataforma de força, baropodometria eletrônica ou PDC. Porém, a FLP, representa um método que contribui para avaliação do controle postural e diagnóstico em portadores de distúrbios do equilíbrio.

\section{Conclusão}

Após a leitura deste estudo, concluiu-se que os resultados apontaram forte correlação entre os métodos de avaliação empregados, e os sujeitos do estudo apresentaram função vestibular normal, de acordo com valores de referência da FLP.

Arealização deste estudo aventura a consolidação de um novo método quali/quantitativo para avaliar o controle postural, e, sobretudo, buscar conhecimento sobre suas restrições e vantagens, servindo de fonte de literatura para estudos futuros que, certamente se farão necessários.

\section{Referências}

BANKOFF, A. D .P.; BEKEDORF, R. G.; SCHMIDT. A.; CIOL, P.; ZAMAI, C. A. Analisis poddometrico de los atletas de levantamiento de peso mediante la técnica vídeo-podometrica. In: CONGRESSO CIENTÍFICO OLÍMPICO, 1., 1992, Málaga. Anais... Málaga: Instituto Andaluz del Deporte, 1992. v. 1, p. 18.

BARELA, J. A. Estratégias de controle em movimentos complexos: ciclo percepção-ação no controle postural. Revista Paulista de Educação Física, São Paulo, supl. 3, p. 79-88, 2000.

CASTAGNO, L. A. A. New method for sensory organization tests: the foam-laser dynamic posturography. Revista Brasileira de Otorrinolaringologia, São Paulo, v. 60, n. 4, p. $287-296,1994$.

DI GRAZIA, R. C. Alterações posturais relacionadas com a disfunção da articulação temporomandibular e seu tratamento. 2003. Dissertação (Mestrado em Educação Física) - Universidade Estadual de Campinas, Campinas.

DUARTE, M.; ZATSIORSKY, V. M. Effects of body lean and visual information on the equilibrium maintenance during stance. Experimental Brain Research, Berlin, v. 146, n. 1, p. 60-69, 2002.

HERDMAN, S. J. Reabilitação vestibular. 2.ed. São Paulo: Manole, 2002.

HORAK, F. B.; MACPHERSON, J. M. Postural orientation and equilibrium. In: ROWELL, L. B.; SHERPHERD, J. T (Ed.) Handbook of physiology: a critical, comprehensive presentation of physiological knowledge and concepts. New York: Oxford American Physiological Society, 1996. p. 255-92.

HORAK, F. B.; NASHNER, L. Central programming of postural movements: adaptation to altered supportsurface configurations. Journal of Neurophysiology, Bethesda, v. 55, n. 6, p. 1369-81, 1996.

HORAK, F. B.; SHUPERT, C. L.; MIRKA, A. Components of postural dyscontrol in the elderly: a review. Neurobiology of Aging, Fayetteville, v. 10, n. 6, p. 727-38, 1989.

LEDIN, T.; ODKVIST, L. M. Dynamic posturography. Acta Awho, São Paulo, v. 12, n. 3, 1993. 
LOBO, M. B. Uma proposta de reabilitação vestibular em grupo para idosas institucionalizadas. 2002. Dissertação (Mestrado em Distúrbios da Comunicação Humana) Universidade Federal de Santa Maria, Santa Maria.

MAKI, B. E.; MCILROY, W. E. Postural control in the oder adult. Clinics in Geriatric Medine, Philadelphia, v. 12, n. 4, p. 635-658, 1996.

NASHNER, L. M. A model describing vestibular detection of body sway motion. Acta Oto-laryngologica, Stockholm, v. 72, n. 6, p. 429-36, 1971.

Computerized dynamic posturography: clinical applications. In: JACOBSON, G. P.; NEWMAN, C. W.; KARTUSH, J. M. The handbook of balance function testing. St. Louis: Mosby-Year Book, 1993. p. 308-333.

SAGE, G. H. Motor learnig and control: a neuropsychological approach. Dubuque: WCB/McGrawHill, 1984.
VIEIRA, G. R. Posturografia dinâmica em indivíduos que trabalham na construção civil exposto à altitude. 2006. Dissertação (Mestrado em Distúrbios da Comunicação Humana) - Universidade Federal de Santa Maria, Santa Maria.

VOORHESS, R. L. The role of dynamic posturography in neurotologic diagnosis. Laryngoscope, Saint Louis, v. 99, n. 10, p. 995-1001, 1989.

WINTER, D. A. Biomechanics and motor control of human movement. New York: Macmillan Publishing Company, 1990.

YASSUE, M. M.; LOTH, E. A.; ROSSI, A. G.; BERTOLINI, G. R. F. Avaliação do equilíbrio em idosos portadores de Diabetes mellitus através de posturografia dinâmica foam-laser. In: CONGRESSO SULBRASILEIRO DE MEDICINA O ESPORTE, 5., 2006, Bento Gonçalves. Anais... Bento Gonçalves: Editora, 2006. e páginas. 